\title{
Oncolytic recombinant herpes simplex virus for treatment of orthotopic liver tumors in nude mice
}

\author{
YOUNG SIK CHUNG ${ }^{1}$, SHIN-ICHI MIYATAKE ${ }^{2}$, AKIKO MIYAMOTO ${ }^{1}$, \\ YOSHIHARU MIYAMOTO ${ }^{1}$, TAKEHIKO DOHI ${ }^{1}$ and NOBUHIKO TANIGAWA ${ }^{1}$ \\ Departments of ${ }^{1}$ General and Gastroenterological Surgery and ${ }^{2}$ Neurosurgery, \\ Osaka Medical College, 2-7 Daigaku-machi, Takatsuki, Osaka 569-8686, Japan
}

Received October 18, 2005; Accepted December 2, 2005

\begin{abstract}
Cell-specific, replicating viruses are being developed as a new class of oncolytic agents. A novel approach to viral gene therapy with the use of replication-competent herpes simplex virus has been described; G92A is a replicationcompetent, multimutant oncolytic herpes simplex virus (HSV) that has been evaluated for anticancer effects and selectivity in the treatment of subcutaneous tumors. G92A replicates efficiently in albumin-producing tumor cell lines but not in non-albumin-producing tumor cell lines, whereas both types are equally susceptible to a non-tissue-specific recombinant $\mathrm{HSV}, \mathrm{hrR} 3$. In this study, we analyzed the antitumoral efficacy of a single intrasplenic G92A or hrR3 injection in nude mice. In vivo, G92A replicated well in liver xenografts of human albumin-producing hepatoma cells (Hep3B) but not in liver xenografts of a non-albumin-producing malignant colon tumor cell line (HT29), whereas hrR3 replicated well in both tumor types. G92A effectively and selectively replicated throughout liver tumors without apparent hepatotoxicity and inhibited tumor growth, leading to a significantly increased survival time. By monitoring lacZ histochemical staining, we determined the oncolytic potential of recombinant HSV against liver tumors. Our results indicate that G92A warrants further investigation as a clinical therapy against malignant liver tumors.
\end{abstract}

\section{Introduction}

Malignant liver tumor is a leading cause of cancer death, accounting for approximately 600,000 cases worldwide in 2000. In Japan, the incidence has increased. The prognosis of untreated malignant liver tumors is poor. For patients with multi-focal tumors localized to the liver, regional approaches

Correspondence to: Dr Nobuhiko Tanigawa, Department of General and Gastroenterological Surgery, Osaka Medical College, 2-7 Daigaku-machi, Takatsuki, Osaka 569-8686, Japan

E-mail: sur001@poh.osaka-med.ac.jp

Key words: recombinant herpes simplex virus, gene therapy, G92A, hrR3, malignant liver tumor such as orthotopic liver transplantation offer long-term survival. However, the supply of livers available for transplantation is very limited. Given the expected number of patients with liver tumor over the next 20 years, development of more effective treatment modalities is urgently needed.

Several gene therapy strategies have been examined for their potential in the treatment of cancer. Tumor-targeted replication-competent viral agents represent a feasible complement to cancer therapy. In addition to adenovirus, herpes simplex virus (HSV), vaccinia virus and reovirus that have been molecularly engineered to replicate specifically within tumor cells, viruses with inherent tumor specificities are actively being developed as oncolytic agents for cancer treatment (1). Compared to other replication-competent oncolytic vectors, $\mathrm{HSV}$ is particularly appealing for its exceptionally rapid replication rate within tumor cells $(8-10 \mathrm{~h})$, such that the oncolytic effects may be manifested maximally before the onset of potentially neutralizing antiviral immune responses in the host. HSV infections in humans are asymptomatic in most cases or result in mild febrile illness. G92A is a secondgeneration replication-conditional HSV-1 mutant that is regulated by the albumin enhancer/promoter (2). G92A efficiently suppressed subcutaneously derived albuminproducing tumor growth in nude mice (3). hrR3 [an HSV-1 strain with a defective ribonucleotide reductase $(R R)$ gene] (4) has also been examined as a replication-competent oncolytic virus because of its preferential replication at low levels in non-neoplastic cells and in vivo virulence at high titer $(5,6)$. The selective replication of viruses bearing mutations of non-essential viral genes can destroy tumor cells. A previous study showed that hrR3 completely eliminates the tumor burden and confers a survival advantage in an intraperitoneal model of pancreatic cancer in athymic mice (7). Toda et al reported that, in vivo, all replication-conditional HSV-1 mutants assayed were effective antitumor agents in experimental models of central nervous system (CNS) tumors and extra-CNS malignancies (8-10). In the present study, we investigated the therapeutic efficacy of G92A and hrR3 in nude mice with malignant liver tumors. We show that intrasplenically inoculated G92A replicated well in liver xenografts of human hepatoma cells (Hep3B) in nude mice but not in human colon carcinoma liver tumors (HT29), whereas hrR3 replicated well in both tumor types. A single dose of the vector was injected intrasplenically into orthotopically implanted malignant 
tumor cell lines in nude mice and led to a significantly prolonged survival. The use of replication-competent viral vectors controlled by cell-specific transcriptional regulatory sequences provides a new strategy for tumor therapy with inherent specificity for replication in tumor cells due to their attenuated antiviral responses. Our results show that G92A and hrR3 are effective oncolytic agents against malignant liver tumors in nude mice. In addition, G92A warrants further development as a selective therapy for patients with malignant liver tumors.

\section{Materials and methods}

Cells and viruses. The albumin-expressing Hep3B human hepatoma cell line, the human HT29 colon carcinoma nonalbumin-expressing tumor cell line and Vero cells (African green monkey kidney cells) were obtained from the American Type Culture Collection (Manassas, VA, USA), and E5 cells (Vero cells stably transfected with ICP4) were provided by Neal DeLuca (University of Pittsburgh School of Medicine, Pittsburgh, PA, USA). Hep3B cells were grown in Dulbecco's modified Eagle's medium (DMEM) supplemented with $10 \%$ heat-inactivated FCS (IFCS) (Hyclone, Logan, UT, USA). HT29 cells were grown in RPMI-1640 medium supplemented with $10 \%$ IFCS. Vero and E5 cells were grown in DMEM supplemented with $10 \%$ newborn calf serum (Hyclone).

The structures of G92A and hrR3 have been described in detail $(2,4)$. hrR3, a non-tissue-specific recombinant HSV defective in RR expression, contains the Escherichia coli lac $Z$ gene inserted into the RR gene locus. The lac $Z$ gene is driven by the ICP6 immediate-early gene promoter. hrR3, kindly provided by Sandy Weller (University of Connecticut Health Center, Farmington, CT, USA), was passaged on
Table I. Treatment of diffuse liver tumors with HT29 and Hep3B.

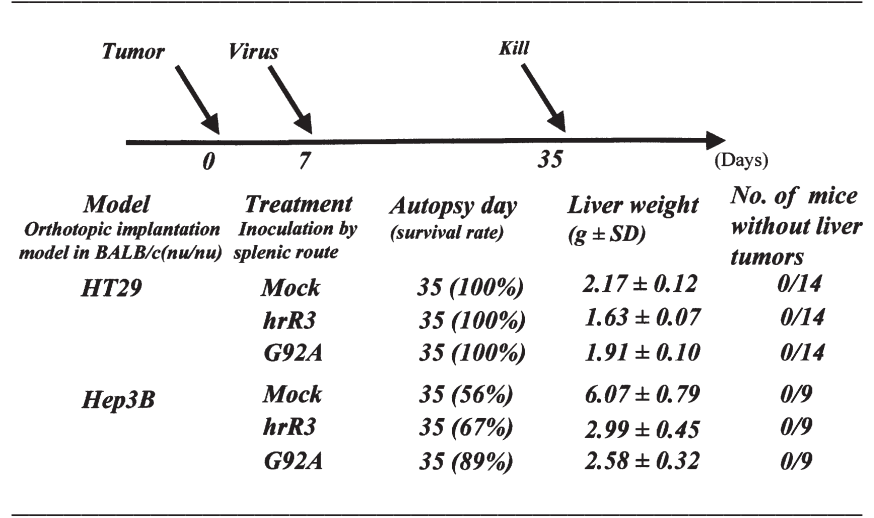

Mice bearing diffuse HT29 and Hep3B liver tumors established as described were treated with $5 \times 10^{6} \mathrm{pfu}$ of G92A or hrR3. For mock injection, the same volume of virus buffer was used. Mice were sacrificed 35 days ater tumor injection, and livers were analyzed.

parental Vero cells and stored at $-80^{\circ} \mathrm{C}$ until use. G92A (parental strain KOS) was generated from E5 cells at low multiplicity of infection (MOI) of E5 cells. The virus was prepared from infected cells by freeze-thaw/sonication, lowspeed centrifugation, ultracentrifugation of supernatant and resuspension of virus pellet in virus buffer $(150 \mathrm{mM} \mathrm{NaCl} /$ 20 mM Tris- $\mathrm{HCl}, \mathrm{pH} 7.5)$.

Cell destruction assay. Hep3B and HT29 cells were plated onto 6-well dishes (Falcon; Becton-Dickinson, Franklin Lakes,

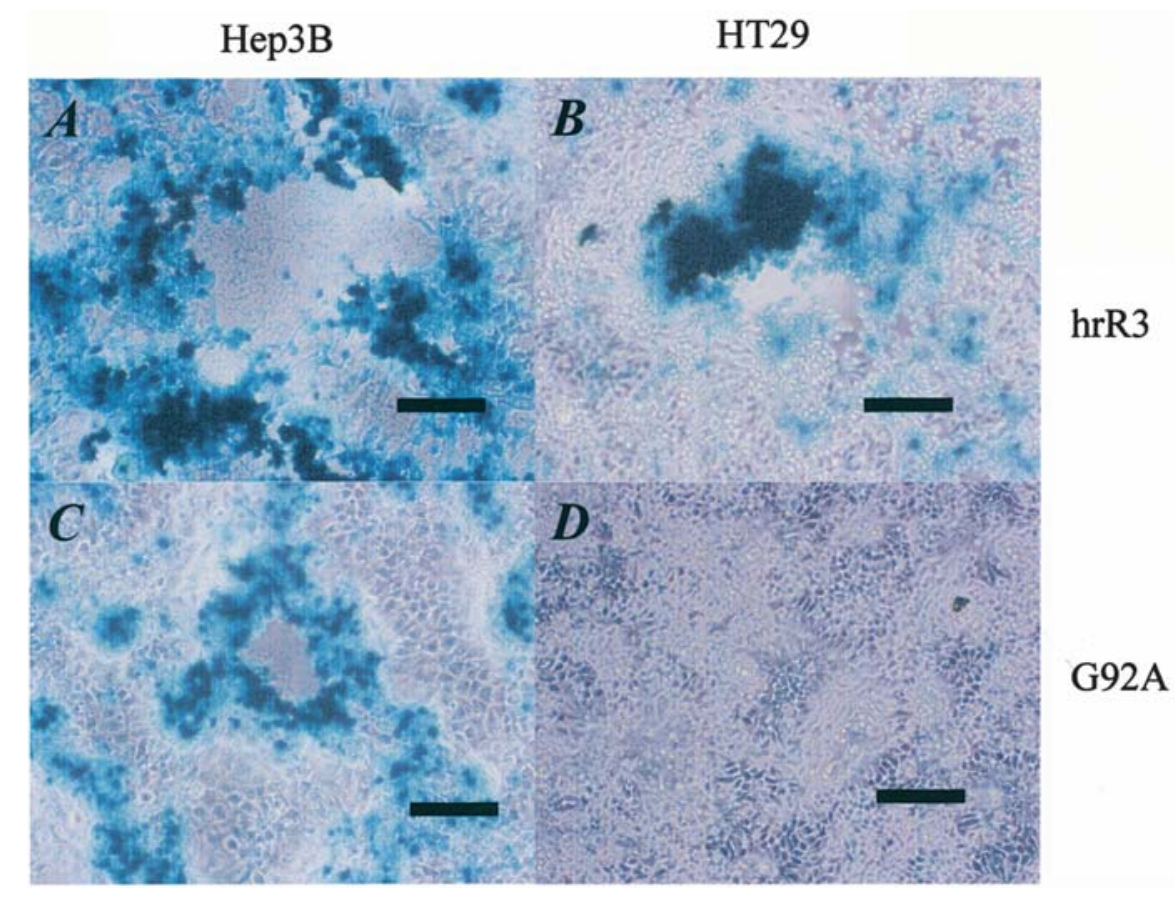

Figure 1. In vitro cell destruction assay. Target cells $\left(1 \times 10^{5}\right)$ of Hep3B (A and C) and HT29 (B and D) were plated onto 6-well dishes 24 h before virus infection. Cells were infected with G92A (C and D) or hrR3 (A and B) at an MOI of 0.07. Infected cells were fixed $72 \mathrm{~h}$ after infection and stained with X-gal (black precipitate). Infection with hrR3 resulted in marked cell destruction irrespective of cell type, whereas infection with G92A resulted in replication and destruction of albumin-expressing tumor cells only. Bars, $0.05 \mathrm{~mm}$. 


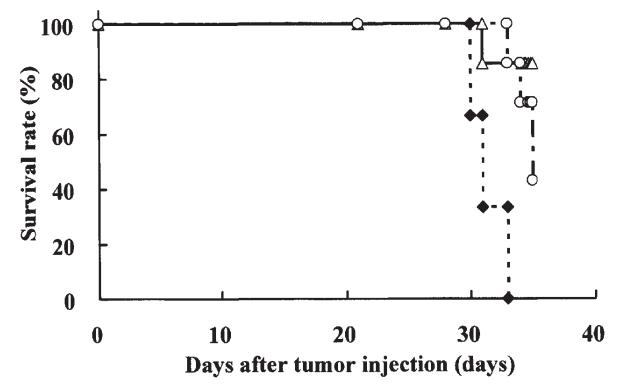

- - Hep3B+buffer $\rightarrow-$ Hep3B+G92A $\rightarrow-$ Hep3B+hrR3

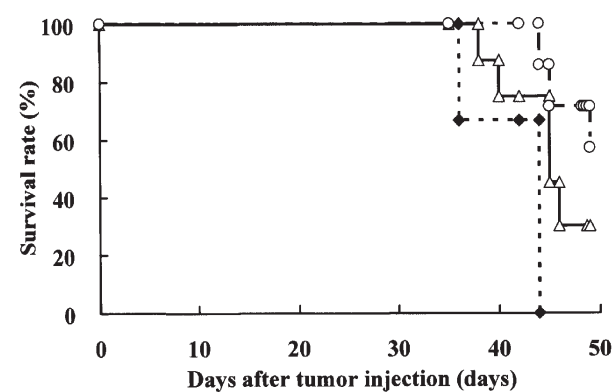

- - HT29+buffer $\neg-$ HT29+G92A $\multimap$ HT29+hrR3

Figure 2. Survival curves of tumor bearing mice infected with G92A or hrR3. Hep3B and HT29 cells $\left(1 \times 10^{5}\right)$ were injected directly into the right hepatic lobe of nude mice. Animals then received intrasplenic treatment on days 7 with either G92A $\left(5 \times 10^{6}\right.$ pfu for Hep3B, n=9, group B; HT29, n=9, group E) or hrR3 (5x106 pfu for Hep3B, n=9, group C; HT29, n=9, group F). Control animals received virus buffer alone (Hep3B, $n=5$, group A; HT29, $\mathrm{n}=5$, group D). Curves represent the sum of two experiments carried out under identical conditions. Survival was evaluated using the Kaplan-Meier method and statistical differences in survival were determined by log-rank analysis. Statistical significance was set at $\mathrm{p}<0.05$. (group A vs group B, $\mathrm{p}<0.05$; group $\mathrm{A}$ vs group $\mathrm{C}, \mathrm{p}<0.05$; group $\mathrm{D}$ vs group $\mathrm{E}$, not significant; group $\mathrm{D}$ vs group $\mathrm{F}, \mathrm{p}<0.05)$.

NJ, USA) 24 h before virus infection. Cells were infected with G92A or hrR3 at an MOI of 0.07 in $0.7 \mathrm{ml}$ phosphatebuffered saline (PBS) supplemented with $1 \%$ IFCS. Virus inoculum was removed after $60 \mathrm{~min}$, and the cells were incubated in DMEM supplemented with $10 \%$ IFCS at $37^{\circ} \mathrm{C}$ in a humidified atmosphere of $5 \% \mathrm{CO}_{2}$. At the indicated times, cells were fixed ( $2 \%$ formaldehyde $/ 0.5 \%$ glutaraldehyde) and stained histochemically in X-gal solution [1 mg/ml 5-bromo4-chloro-3-indolyl-bD-galactopyranoside (X-gal), $5 \mathrm{mM}$ $\left.\mathrm{K}_{3}\left[\mathrm{Fe}(\mathrm{CN})_{6}\right], 5 \mathrm{mM} \mathrm{K}_{4} \mathrm{Fe}(\mathrm{CN})_{6} \cdot 3 \mathrm{H}_{2} \mathrm{O}, 2 \mathrm{mM} \mathrm{MgCl}{ }_{2}\right]$ at $37^{\circ} \mathrm{C}$ for several hours.

\section{Animal studies}

Hepatic tumor model and treatment with HSV vector. All procedures involving animals were approved by the guidelines of the Institutional Animal Care and Use Committee of Osaka Medical College. Pathogen-free, 4- to 5-week-old female $\mathrm{BALB} / \mathrm{c}$ nude mice were allowed to acclimate for 1 week. Mice were anesthetized with a $0.35-\mathrm{ml}$ i.p. injection of $84 \%$ bacteriostatic saline, $10 \%$ pentobarbital $(1 \mathrm{mg} / \mathrm{kg}$; Abbott Laboratories, Chicago, IL, USA) and 6\% ethyl alcohol for all surgical procedures. For liver tumor formation, a $1-\mathrm{cm}$ subcostal incision was made to divide the peritoneum and expose the liver. Mice received an orthotopic implant of $1 \times 10^{5}$ heterogeneic Hep3B or HT29 cells in a 50- $\mu 1$ singlecell suspension injected directly into the right hepatic lobe with a Hamilton syringe (Reno, NV, USA) and $30 \mathrm{G}$ needle. One week later, $5 \times 10^{6}$ plaque-forming units (pfu) of G92A or
hrR3 in 50- $\mu 1$ virus buffer was injected into the spleen, or an equivalent volume of virus buffer alone was injected as a control. To assess tumor response and virus efficacy, animals were sacrificed 35 days after tumor injection, and livers were removed for examination (Table I). Data were analyzed using the SPSS statistical software package. In addition, groups of animals were followed up for survival. Survival was evaluated using the Kaplan-Meier method and log-rank test. Statistical significance was set at $\mathrm{p}<0.05$.

Liver sections and histochemical staining. To analyze liver tumors and liver tissue for the presence of rHSV, direct injection of $1 \times 10^{5}$ Hep3B or HT29 cells was performed during laparotomy. Intrasplenic rHSV injections were performed 2 weeks after tumor implantation, and animals were sacrificed 3 days after virus challenge. Livers were resected after a PBS flush and fixed with $2 \%$ paraformaldehyde, $5 \mathrm{mM}$ Ethylene Glycol Bis ( $\left(\right.$-aminoethylether)- $N, N, N^{\prime}, N^{\prime}$-tetraacetic acid and $2 \mathrm{mM} \mathrm{MgCl}_{2}$ in $0.1 \mathrm{M}$ Pipes buffer (pH 7.3) overnight. Whole livers were cut into $20-\mu \mathrm{m}$ sections by cryostat and stained histochemically in X-gal solution with $0.02 \%$ NP-40 and $0.01 \%$ sodium deoxycholate. Paraffin-embedded sections were subjected to hematoxylin-and-eosin staining for histological analysis.

\section{Results}

In vitro oncolytic activities of G92A and hrR3. The G92A and hrR3 viruses were replicated in E5 and Vero cells as described in Materials and methods. The cytotoxic ability of both recombinant viruses was then examined. Hep3B and HT29 cells $\left(1 \times 10^{5}\right)$ were plated onto 6 -well dishes $24 \mathrm{~h}$ before virus infection. To assess whether these viruses have the ability to preferentially replicate and kill malignant tumor cells, cells were examined for cytopathic effects, and viral replication was analyzed by lacZ expression with $\mathrm{X}$-gal staining. Infection with G92A or hrR3 was as follows: Hep3B (Fig. 1A and C) and HT29 (Fig. 1B and D) cells were infected at an MOI of 0.07 with G92A (Fig. 1C and D) or hrR3 (Fig. 1A and B). Plaque formation was observed $72 \mathrm{~h}$ after infection; infected cells were fixed and stained with X-gal. The morphology of non-albumin-producing HT29 cells infected with hrR3 showed typical HSV cytopathic effects (Fig. 1B), whereas those infected with G92A were unaltered (Fig. 1D). Albuminproducing Hep3B cells (Fig. 1A and C) became non-viable and completely floated off the tissue culture plates in response to infection with rHSV. hrR3 infection showed marked cell destruction irrespective of cell type, whereas G92A infection showed replication and destruction only in albumin-expressing tumor cells (Hep3B).

In vivo target cell specificity. To investigate the potential of G92A and hrR3 for use in therapy of malignant tumors, we tested the viruses in orthotopic liver tumors in nude mice. To produce diffuse liver tumors, $1 \times 10^{5}$ Hep3B cells and HT29 cells was injected directly into the right hepatic lobe of 9 and 14 nude mice, respectively. After 7 days, $5 \times 10^{6}$ pfu of G92A or hrR3 was injected intrasplenically. The animals were sacrificed 35 days after tumor injection for measurement of liver weight. Results indicated that albumin-producing tumor growth was 

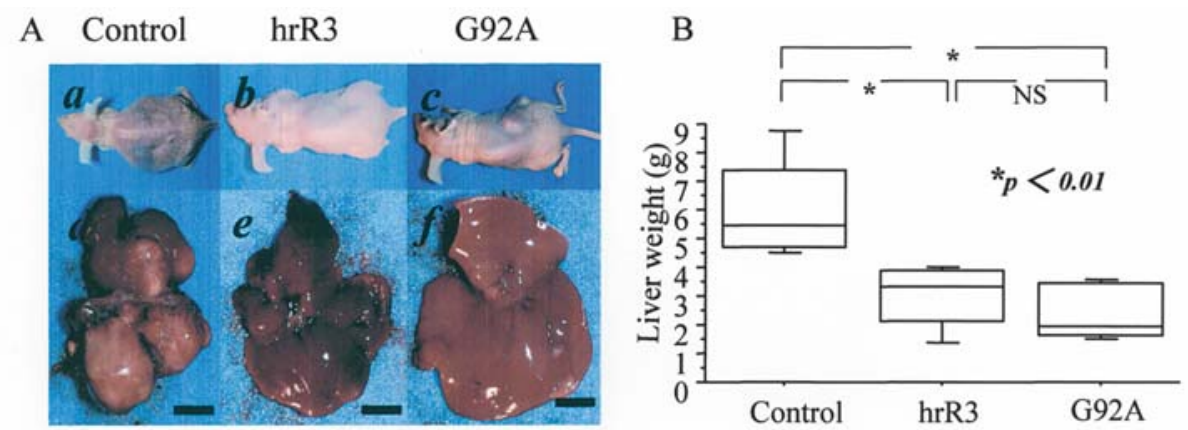

Figure 3. Comparison of G92A and hrR3 viral therapy in Hep3B liver tumors. A, mice (a-c) treated with a single intrasplenic injection of buffer (a and d), hrR3 (5x10 $\mathrm{pfu}$ ) (b and e) or G92A (c and f) and livers (d-f). G92A- and hrR3-injected mice showed significant differences compared to buffer-injected mice. Bars, $5 \mathrm{~mm}$. B, tumor burdens were assessed by weight. Mice were sacrificed 35 days after tumor injection, and livers were analyzed. $\mathrm{p}<0.01$ for hrR3 versus control and for G92A versus control; $\mathrm{p}=0.32$ for hrR3 versus G92A. NS, not significant.

A

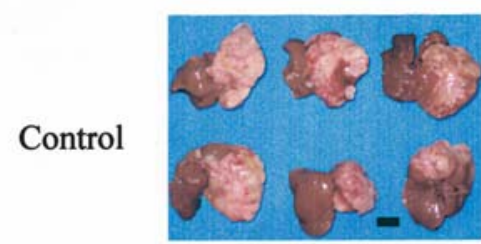

G92A
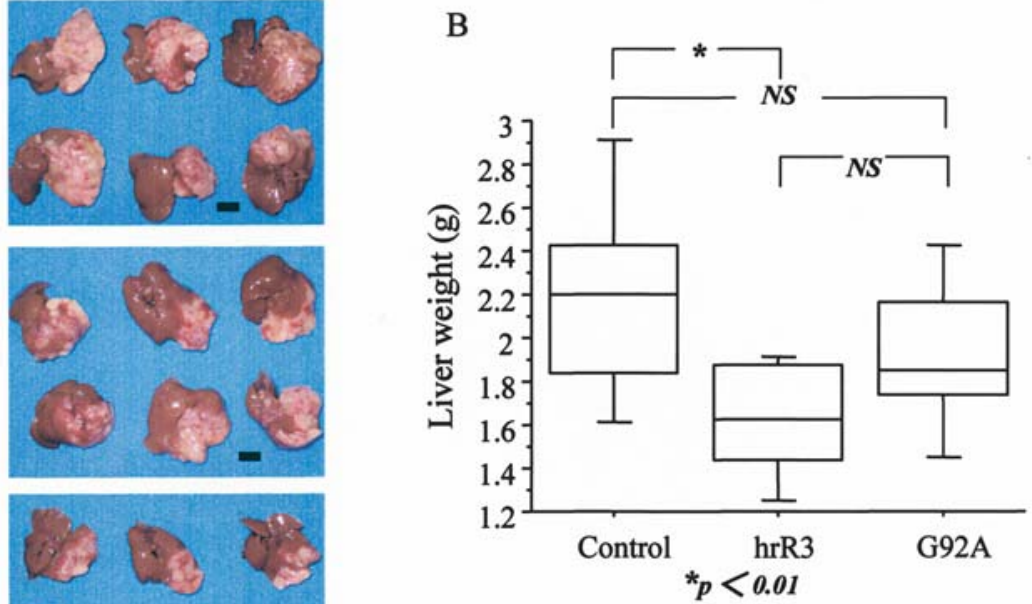

hrR3

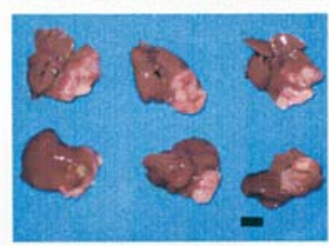

Figure 4. Comparison of G92A and hrR3 viral therapy in HT29 liver tumors. A, HT29 liver tumors treated with 5x106 pfu of G92A or hrR3. Mice were sacrificed 35 days after tumor injection, and livers were analyzed. Representative livers from mice treated with a single intrasplenic injection of buffer (control), hrR3 or G92A are shown. hrR3-injected mice showed significant differences compared to buffer-injected mice. G92A-injected mice showed no remarkable differences compared to buffer-injected mice. Bars, $5 \mathrm{~mm}$. B, tumor burden was assessed by weight. Mice were sacrificed 35 days after tumor injection, and livers were analyzed. $\mathrm{p}<0.01$ for hrR3 versus control; there was no significant difference for G92A versus control or for hrR3 versus G92A. NS, not significant.

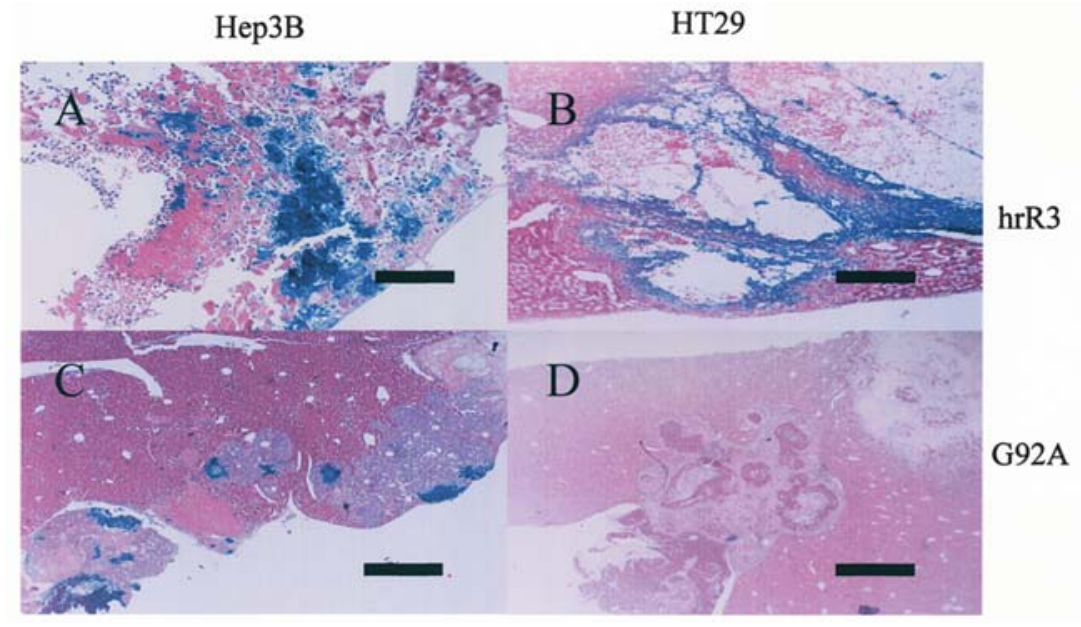

Figure 5. Microscopic visualization of virus spread. Tumors were inoculated with $5 \times 10^{6} \mathrm{pfu}$ of $\mathrm{G} 92 \mathrm{~A}(\mathrm{C}$ and $\mathrm{D})$ or hrR3 (A and B). Three days after virus inoculation, livers were removed after PBS flush and stained histochemically with X-gal. G92A spread well in Hep3B tumor nodules (C) but not at all in HT29 tumor nodules (D). hrR3 showed effective viral spread in Hep3B and in HT29 tumor nodules (A and B). Bars, 0.5 mm. 
significantly inhibited by G92A or hrR3 injection compared to that in control animals injected with injection buffer alone, whereas non-albumin-producing tumor growth was inhibited only by hrR3 injection (Figs. 3 and 4). Differences in survival were statistically significant in Hep3B-injected mice between those untreated and those treated with hrR3 or G92A at 35 days after Hep3B injection ( $\mathrm{p}<0.05$ by log-rank test). Whereas, there was significant difference in survival between untreated HT29injected mice and those treated only with hrR3 compared with those treated with G92A at 49 days after HT29 injection ( $<<0.05$ by log-rank test) (Fig. 2).

Hep3B injection models were monitored for 35 days. None of the mice receiving viral therapy developed neurological symptoms or other adverse effects. Results of G92A and hrR3 viral therapies in Hep3B liver tumors are shown in Fig. 3A. Representative mice and livers after treatment with a single intrasplenic injection of virus buffer alone (a and d), hrR3 (b and e) or G92A (c and f) are shown. Mice were sacrificed 35 days after tumor injection, and livers were analyzed (Fig. 3B). A total of $5 \times 10^{6}$ pfu of G92A or hrR3 showed significant differences compared to buffer injection. Tumor burden was assessed by weight. The control group $(\mathrm{n}=9)$ had a mean weight of $6.07 \mathrm{~g}$, the hrR3-treated group $(\mathrm{n}=9)$ had a mean weight of $2.99 \mathrm{~g}(\mathrm{p}<0.01$; unpaired t-test) and the G92A-treated group ( $\mathrm{n}=9$ ) had a mean weight of $2.58 \mathrm{~g}$ ( $\mathrm{p}<0.01$; unpaired t-test). HT29 liver tumors treated with $5 \times 10^{6}$ pfu of G92A or hrR3 are shown in Fig. 4A. Mice were sacrificed 35 days after tumor injection, and livers were analyzed. Livers from mice treated with a single intrasplenic injection of buffer (a), hrR3 (b) or G92A (c) are shown. hrR3injected mice showed significant differences compared to buffer-injected mice. G92A-injected mice showed no remarkable differences compared to buffer-injected mice. G92A and hrR3 viral therapy for HT29 liver tumors was also assessed by weight (Fig. 4B). The control group $(n=14)$ had a mean weight of $2.17 \mathrm{~g}$, the hrR3-treated group $(\mathrm{n}=14)$ had a mean weight of $1.63 \mathrm{~g}(\mathrm{p}<0.01$; unpaired t-test) and the G92A-treated group $(n=14)$ had a mean weight of $1.91 \mathrm{~g}$ (no significant difference; unpaired t-test).

We next sought to determine whether G92A or hrR3 replicates selectively in diffuse malignant liver tumors. To produce diffuse liver tumors, $1 \times 10^{5}$ Hep3B or HT29 cells was injected into the right hepatic lobe of nude mice. Thirtythree days after tumor injection, $5 \times 10^{6}$ pfu of G92A or hrR3 was injected intrasplenically. Animals were sacrificed 3 days after virus inoculation, livers were removed after a PBS flush and sectioned, and sections were stained with $\mathrm{X}$-gal for examination of lacZ gene expression. G92A infection spread well in Hep3B tumor nodules (Fig. 5C) and not at all in HT29 tumor nodules and normal liver (Fig. 5D). hrR3 showed effective viral spread in Hep3B and HT29 tumor nodules with partial spread in normal liver (Fig. 5A and B). Spleen, intestine and kidney specimens from the same mice showed no lacZ staining (data not shown). Mice injected with virus buffer showed no lacZ staining (data not shown).

\section{Discussion}

Increased understanding of the factors responsible for HSV virulence and replication has revealed a potential role of
HSV mutants in treating malignancies (11). Neurological tumors were first targeted due to natural HSV neurotropism. Various recombinant, replicating HSV mutants have since been tested as therapies for extra-CNS malignancies $(5,10,12)$. Varghese et al reported the safety and effectiveness of rHSV 1716, harboring a homozygous deletion of ICP34.5, against human malignant mesothelioma cell lines in vitro and in a mouse model of epithelial ovarian cancer (13-15). The rHSV mutant G207 has a deletion in ICP34.5 and a lacZ gene insertion inactivating the ICP6 gene (8). Both rHSV 1716 and G207 were effective in animal models of malignant peritoneal disease induced by injection of human ovarian cancer cells $(14,16)$. Intraperitoneal administration of G207 was also reported to have therapeutic potential against peritoneal tumors (17). rHSV R3616, an ICP34.5 deficient, replication-restricted HSV-1 was equally effective against chemotherapy-sensitive and -resistant ovarian cancer cells in vitro and in vivo (16). Thus, rHSV mutants may be effective in the treatment of malignant tumors. Despite promising findings, few phase 1 or 2 studies have been reported $(18,19)$. Our study examined the effects of two types of HSV mutant on malignant liver tumor cells in nude mice. G92A and hrR3 prolonged animal survival and appeared to significantly effect tumor regression in albumin-producing tumors (Hep3B), whereas G92A had no effect on non-albumin-producing tumors (HT29), indicating that the oncolytic effect of G92A is more selective than that of hrR3.

The precise mechanism of viral cytotoxicity remains to be elucidated. The antitumor effect of rHSV is reported to be much greater than its evident distribution throughout tumors (20-25). HSV infection induces apoptosis in adjacent, uninfected cells in vitro and in vivo (26). The route of administration will be a critical determining factor in the efficacy of viral therapy. At first, we examined the efficacy of intraportal delivery of hrR3 via splenic injection. The efficacy and specificity of $l a c Z$ gene expression in tumor cells were striking. The level of lacZ expression in tumor nodules was extremely high compared to that in normal hepatic parenchyma. Very low incidence but distinct blue-stained cells were observed in normal liver by hrR3, whereas no blue staining of cells was observed in normal livers by the same delivery of G92A (data not shown). Thus, hrR3 also has the possibility of spreading in normal liver. As expected, G92A is more safe and has more feasibility of therapeutic strategy. Further studies are warranted to assess the level of lacZ expression at various time intervals after infection and to examine the immune responses to G92A and hrR3 when they are injected into the spleen. In the present study, we observed efficient rHSV vector infection and replication in human hepatoma (Hep3B) and colon cancer (HT29) cell lines. In contrast, normal mouse hepatocytes are resistant not only to virus infection but also to replication. A single intrasplenic injection of rHSV into malignant liver tumors showed potent antitumor activity. The ability of rHSV to replicate intratumorally in vivo was shown by X-gal staining of lacZ $(5,27-$ 29). Our data indicate that intratumoral HSV replication led to slower tumor growth, tumor destruction and prolonged survival. These results are consistent with those reported previously for subcutaneous injections of rHSV against malignant tumors in nude mice (3). There was presumably no 
uncontrolled viral replication in treated animals because it has been reported that viral replication is limited to the tumor bed and decreased to negligible levels after 7 days $(8,30,31)$. We did not observe any adverse effects of virus in animals treated with the indicated vector doses. In conclusion, HSV replication is tumor-selective in vitro as well as in vivo and produces significant destruction of large tumors before the onset of potentially neutralizing antiviral immune responses in the host. A single intrasplenic injection of virus can significantly prolong the survival of tumor-bearing animals. This oncolytic virus system warrants further development as a therapeutic option for clinical treatment of malignant liver tumors.

\section{References}

1. Vile RG, Diaz RM, Miller N, et al: Tissue-specific gene expression from Mo-MLV retroviral vectors with hybrid LTRs containing the murine tyrosinase enhancer/promoter. Virology 214: 307-313, 1995.

2. Miyatake S, Iyer A, Martuza RL, et al: Transcriptional targeting of herpes simplex virus for cell-specific replication. J Virol 71: 5124-5132, 1997.

3. Miyatake SI, Tani S, Feigenbaum F, et al: Hepatoma-specific antitumor activity of an albumin enhancer/promoter regulated herpes simplex virus in vivo. Gene Ther 6: 564-572, 1999.

4. Goldstein DJ and Weller SK: Factor(s) present in herpes simplex virus type 1-infected cells can compensate for the loss of the large subunit of the viral ribonucleotide reductase: characterization of an ICP6 deletion mutant. Virology 166: 41-51, 1988.

5. Carroll NM, Chiocca EA, Takahashi K, et al: Enhancement of gene therapy specificity for diffuse colon carcinoma liver metastases with recombinant herpes simplex virus. Ann Surg 224: 323-330, 1996.

6. Kramm CM, Chase M, Herrlinger U, et al: Therapeutic efficiency and safety of a second-generation replicationconditional HSV1 vector for brain tumor gene therapy. Hum Gene Ther 8: 2057-2068, 1997.

7. Kasuya H, Nishiyama Y, Nomoto S, et al: Intraperitoneal delivery of hrR3 and ganciclovir prolongs survival in mice with disseminated pancreatic cancer. J Surg Oncol 72: 136-141, 1999.

8. Mineta T, Rabkin SD, Yazaki T, et al: Attenuated multimutated herpes simplex virus- 1 for the treatment of malignant gliomas. Nat Med 1: 938-943, 1995.

9. Yazaki T, Manz HJ, Rabkin SD, et al: Treatment of human malignant meningiomas by G207, a replication-competent multimutated herpes simplex virus 1. Cancer Res 55: 4752-4756, 1995.

10. Toda M, Martuza RL, Kojima $\mathrm{H}$, et al: In situ cancer vaccination: an IL-12 defective vector/replication-competent herpes simplex virus combination induces local and systemic antitumor activity. J Immunol 160: 4457-4464, 1998.

11. Sundaresan P, Hunter WD, Martuza RL, et al: Attenuated, replication-competent herpes simplex virus type 1 mutant G207: safety evaluation in mice. J Virol 74: 3832-3841, 2000.

12. Yoon SS, Carroll NM, Chiocca EA, et al: Cancer gene therapy using a replication-competent herpes simplex virus type 1 vector. Ann Surg 228: 366-374, 1998.

13. Kucharczuk JC, Randazzo B, Chang MY, et al: Use of a 'replication-restricted' herpes virus to treat experimental human malignant mesothelioma. Cancer Res 57: 466-471, 1997.
14. Coukos G, Makrigiannakis A, Kang EH, et al: Use of carrier cells to deliver a replication-selective herpes simplex virus-1 mutant for the intraperitoneal therapy of epithelial ovarian cancer. Clin Cancer Res 5: 1523-1537, 1999.

15. Varghese S and Rabkin SD: Oncolytic herpes simplex virus vectors for cancer virotherapy. Cancer Gene Ther 9: 967-978, 2002.

16. Coukos G, Makrigiannakis A, Kang EH, et al: Oncolytic herpes simplex virus-1 lacking ICP34.5 induces p53-independent death and is efficacious against chemotherapy-resistant ovarian cancer. Clin Cancer Res 6: 3342-3353, 2000.

17. Bennett JJ, Delman KA, Burt BM, et al: Comparison of safety, delivery, and efficacy of two oncolytic herpes viruses (G207 and NV1020) for peritoneal cancer. Cancer Gene Ther 9: 935-945, 2002.

18. Markert JM, Medlock MD, Rabkin SD, et al: Conditionally replicating herpes simplex virus mutant, G207 for the treatment of malignant glioma: results of a phase I trial. Gene Ther 7: 867-874, 2000 .

19. Kasuya H, Takeda S, Nomoto S, et al: The potential of oncolytic virus therapy for pancreatic cancer. Cancer Gene Ther 12: 725-736, 2005.

20. Kooby DA, Carew JF, Halterman MW, et al: Oncolytic viral therapy for human colorectal cancer and liver metastases using a multi-mutated herpes simplex virus type-1 (G207). FASEB J 13: $1325-1334,1999$.

21. Yoon SS, Nakamura H, Carroll NM, et al: An oncolytic herpes simplex virus type 1 selectively destroys diffuse liver metastases from colon carcinoma. FASEB J 14: 301-311, 2000.

22. Bennett JJ, Kooby DA, Delman K, et al: Antitumor efficacy of regional oncolytic viral therapy for peritoneally disseminated cancer. J Mol Med 78: 166-174, 2000.

23. Bennett JJ, Malhotra S, Wong RJ, et al: Interleukin 12 secretion enhances antitumor efficacy of oncolytic herpes simplex viral therapy for colorectal cancer. Ann Surg 233: 819-826, 2001.

24. Bennett JJ, Tjuvajev J, Johnson P, et al: Positron emission tomography imaging for herpes virus infection: Implications for oncolytic viral treatments of cancer. Nat Med 7: 859-863, 2001.

25. Iizuka Y, Suzuki A, Kawakami Y, et al: Augmentation of antitumor immune responses by multiple intratumoral inoculations of replication-conditional HSV and interleukin-12. J Immunother 27: 92-98, 2004.

26. Stanziale SF, Petrowsky H, Adusumilli PS, et al: Infection with oncolytic herpes simplex virus-1 induces apoptosis in neighboring human cancer cells: a potential target to increase anticancer activity. Clin Cancer Res 10: 3225-3232, 2004.

27. Boviatsis EJ, Chase M, Wei MX, et al: Gene transfer into experimental brain tumors mediated by adenovirus, herpes simplex virus, and retrovirus vectors. Hum Gene Ther 5: 183-191, 1994.

28. Boviatsis EJ, Scharf JM, Chase M, et al: Antitumor activity and reporter gene transfer into rat brain neoplasms inoculated with herpes simplex virus vectors defective in thymidine kinase or ribonucleotide reductase. Gene Ther 1: 323-331, 1994.

29. Miyatake S: Gene therapy using tissue-specific replication competent HSV. Hum Cell 15: 130-137, 2002.

30. Krisky DM, Marconi PC, Oligino TJ, et al: Development of herpes simplex virus replication-defective multigene vectors for combination gene therapy applications. Gene Ther 5: 1517-1530, 1998.

31. Krisky DM, Wolfe D, Goins WF, et al: Deletion of multiple immediate-early genes from herpes simplex virus reduces cytotoxicity and permits long-term gene expression in neurons. Gene Ther 5: 1593-1603, 1998. 